\title{
Evaluation of the effectiveness of a care program for elderly patients with hip fractures: a network strategy
}

Fatima Izabel Dornelles Farias ${ }^{1}$ Newton Luiz Terra' Marcelo Teodoro Ezequiel Guerra²

\section{Abstract}

Objective: Evaluate the effectiveness of a care program for elderly persons with hip fractures due to a fall, based on a public network in Canoas, Rio Grande do Sul, Brazil. Methods: a prospective cohort study of quantitative character was carried out. A total of 182 elderly person above 60 years diagnosed with hip fractures were included. The sample was divided into two groups, one who underwent their usual treatment $(n=91)$ and another who were included in a care program for elderly persons with hip fractures $(\mathrm{n}=91)$. The program had as its main axis a clinical Protocol and an Access Protocol. The treatment of choice was surgical in all cases. In statistical analysis, categorical variables were described by absolute and relative frequencies. The Mann-Whitney test was used to compare numeric variables. In the comparison of proportions, the Pearson's Chi-squared test or Fisher's exact test were applied. The significance level adopted was 5\%. Results: The average age was 79.4 years, with a prevalence of the female gender. The most frequent morbidity was hypertension. After the implementation of the program there was a reduction in the average time between the fracture and the beginning of surgery, the average length of hospitalization, the urinary tract infection rate, the death rate and care costs. Conclusion: The care program of elderly persons with hip fractures modified the expected results as it reduced mortality, average hospital stay, postoperative complications and the costs of treatment during the hospitalization.

Pontifícia Universidade Católica do Rio Grande do Sul (PUCRS), Instituto de Geriatria e Gerontologia (IGG). Porto Alegre, Rio Grande do Sul, Brasil.

2 Universidade Luterana do Brasil (ULBRA), Hospital Universitário de Canoas, Serviço de Ortopedia e Traumatologia (SOT), Canoas, Rio Grande do Sul, Brasil.

Keywords: Elderly. Hip Fractures. Health Services for the Aged. Falls. 


\section{INTRODUCTION}

According to data from the Instituto Brasileiro de Geografia e Estatística (the Brazilian Institute of Geography and Statistics) (IBGE) ${ }^{1}$, serious diseases are common and frequent among the elderly, leading such patients to functional decline, morbidity and mortality. Among these diseases is an acute event of significant proportions, the hip fracture (HF).

For Auerbach et $a .^{2}$, a HF in the elderly is the result of low-impact trauma, most often due to falls, which are increasingly likely as people get older. One in three people over 65 will fall every year, according to epidemiological data from the IBGE ${ }^{1}$. Tsang and Cromwell ${ }^{3}$ state that among the most prevalent risk factors for hip fractures among the elderly are osteoporosis, diabetes, vision problems, inadequate safety/surveillance at home and other health conditions that impair balance.

According to Eriksson et al. ${ }^{4}$ elderly patients with HF have a higher risk of mortality, are unable to return to their previous living conditions, have an increased need for care and supervision, reduced mobility and locomotion capacity, and a greater chance of secondary fractures.

When the costs of treatment are evaluated, Friedman et al. ${ }^{5}$ calculated that in general caring for a hip fracture is three to seven times more expensive than the average cost of other types of treatment. According to Burgers et al. ${ }^{6}$ the elderly spend more time in hospital than other age groups and have a greater exposure to risk factors and more adverse effects arising from the interventions used.

According to data from the British Orthopaedic Association (BOA) ${ }^{7}$, despite the complexity involved in the treatment of $\mathrm{HF}$, there is still significant variation in the key aspects of treatment between hospitals, resulting in considerable differences in the patient experience, especially in relation to the preoperative (in the time patients are referred for treatment) and postoperative (in the care for risk prevention - the risk of developing pressure ulcers, delirium, thromboembolism and infection, among others) periods.

The studies by Mendelson and Friedman ${ }^{8}$ indicate that dedicated programs for elderly persons with
HF can positively to treatment, bringing results with greater overall value. According to Porter and Teisberg ${ }^{9}$, these programs presuppose, in addition to the incorporation of clinical guidelines, the use of a systematic method to achieve adherence, which includes the training of professionals, the nomination of medical and nursing leaders, the monitoring of the units involved, the use of indicators and the reorganization of service logistics and networks.

However, the British Orthopaedic Association ${ }^{7}$ warns that for these practices to be modified, new mechanisms need to be adopted for the coordination of activities, with the search for more collaborative ventures and more precisely executed processes. These variables should be seen as a challenge, and initiatives to improve care quality should be integrated and conducted throughout the care network.

In this context, the present study evaluated the results of a program of care for the elderly with hip fractures caused to falls, applied in a public care network in the city of Canoas, in Rio Grande do Sul.

\section{METHOD}

The research strategy chosen was a cohort study which was retrospective for the control group. It was performed based on the analysis of the medical records, and was prospective for the cases included in the program. Elderly patients of both genders, aged over 60 years, with a diagnosis of $\mathrm{HF}$ due to a fall, were included. HF were considered to be fractures of the proximal end of the femur. All the patients were attended at an Emergency Hospital and transferred for definitive surgical treatment at a hospital in the rear, the Hospital Universitário (HU), both of which were in the town of Canoas in Rio Grande do Sul, Brazil. The Guidelines of the British Association of Orthopaedics ${ }^{7}$ were used as a reference for the sample calculation. Based on a significance level of $5 \%$, a power of $80 \%$, an estimated mortality incidence of $20 \%$ and a $15 \%$ reduction when applied to the protocol, a minimum total of 88 patients in each group was obtained. All patient records in the period were considered eligible and the sample was divided into two groups. The first group of patients was referred to as the usual treatment group ( $\mathrm{n}=91)$ and were admitted to the hospital from June 2014 to June 2015. The second group of elderly patients 
( $\mathrm{n}=91)$ was included in a program with broad clinical guidelines and an access procedure applied in a municipal network that involved multidisciplinary prehospital care teams, a first aid hospital and a back hospital. The main thrust of the program was to prepare people and resources for the early surgical treatment of low-impact HF patients. This group was admitted to the hospital from October 2015 to October 2016.

The demographic data collected were gender and age. Previously investigated morbidities were diabetes mellitus type II, systemic arterial hypertension, dyslipidemia, previous coronary artery disease, congestive heart failure, chronic obstructive pulmonary disease, and chronic renal insufficiency.

To follow up on the results of the program, some indicators were defined as process and outcomes and were then compared with the results of the usual treatment group. The indicators chosen were: use of prophylactic antibiotics, daily evaluation by hospital doctor and orthopedic surgeon by 2 $\mathrm{pm}$, pain evaluation, nutritional assessment, social assessment, planning of discharge from the first day, early withdrawal of a urinary catheter, time elapsed between fracture and beginning of surgery (2-day goal), mean length of hospital stay; 30 -day readmission rate, morbidity rate; mortality rate; rate of surgical site infections, postoperative infection rate; mechanical retention rate, postoperative complication rate - deep venous thrombosis, pulmonary thromboembolism, surgical site infection, pneumonia, urinary tract infection, acute renal failure, stroke, acute myocardial infarction, gastrointestinal bleeding, pressure ulcers, adverse events reported, delirium.

For the composition of costs, three accounting appropriation groups were created - direct costs, daily costs and medical fees. The direct costs included all costs directly related to patient care: emergency room, surgical procedure, medical and nursing services, materials, medicines and prostheses. Daily stay costs included hospitalization, sanitation and food costs. Indirect administrative overheads or transaction costs were not included.

The costs analyzed were calculated by patient and then grouped into mean, median, and total cost, all expressed in reais ( $\mathrm{R} \$$ ) and converted into US dollars based on the average exchange rate in June 2016 (US\$3.42). The cost data were taken from the hospital management tools.

For statistical analysis, the categorical variables related to the morbidity profile were described in absolute and relative frequencies. In order to compare the numerical variables between the group who began surgery in up to 48 hours (two days) and those with a waiting time greater than 48 hours, the Mann-Whitney test was used. In the comparison of proportions, Pearson's chi-square or Fisher's exact tests were applied. For the polytomic variables (more than two categories), the adjusted residuals test was used to locate the significant differences indicated by the chi-square test. The level of significance was $5 \%(p<0.05)$.

The study was approved by the Ethics Research Committee of the Pontifícia Universidade Católica do Rio Grande do Sul (Pontifical Catholic University of Rio Grande do Sul) (PUCRS), CAAE 51213715.5.0000.5336.

\section{RESULTS}

When the profile of the elderly was characterized (table 1) there was a higher prevalence of individuals in the age range between 70 and 90 years $(72.5 \%)$, with a prevalence of the female gender (74.2). 
Table 1. Characterization of profile of patients involved in the study. Porto Alegre, Rio Grande do Sul, 2016.

\begin{tabular}{|c|c|c|c|c|}
\hline \multirow[t]{2}{*}{$\overline{\text { Variables }}$} & $\begin{array}{l}\text { Total } \\
(n=182)\end{array}$ & $\begin{array}{l}\text { Usual treatment } \\
(n=91)\end{array}$ & $\begin{array}{l}\text { Included in the program } \\
(n=91)\end{array}$ & \multirow[t]{2}{*}{$p$} \\
\hline & $n(\%)$ & $n(\%)$ & $n(\%)$ & \\
\hline Gender & & & & 1.000 \\
\hline Female & $135(74.2)$ & $68(74.7)$ & $67(73.6)$ & \\
\hline Male & $47(25.8)$ & $23(25.3)$ & $24(26.4)$ & \\
\hline Age (years) & $79.4( \pm 8.8)$ & $78.7( \pm 8.5)$ & $80.1( \pm 9.0)$ & 0.289 \\
\hline Age range & & & & 0.553 \\
\hline 60 to 70 & $29(15.9)$ & $15(16.5)$ & $14(15.4)$ & \\
\hline 71 to 80 & $67(36.8)$ & $37(40.7)$ & $30(33.0)$ & \\
\hline 81 to 90 & $65(35.7)$ & $31(34.1)$ & $34(37.4)$ & \\
\hline$>90$ & $21(11.5)$ & $8(8.8)$ & $13(14.3)$ & \\
\hline Presence of Morbidities & $159(87.4)$ & $80(87.9)$ & $79(86.8)$ & 1.000 \\
\hline Diabetes mellitus & $42(23.1)$ & $28(30.8)$ & $14(15.4)$ & 0.022 \\
\hline Systemic Arterial Hypertension & $132(72.5)$ & $69(75.8)$ & $63(69.2)$ & 0.406 \\
\hline Dyslipidemia & $18(9.9)$ & $11(12.1)$ & $7(7.7)$ & 0.456 \\
\hline Known Arterial Disease & $41(22.5)$ & $23(25.3)$ & $18(19.8)$ & 0.478 \\
\hline Cardiopulmonary resuscitation & $1(0.5)$ & $1(1.1)$ & $0(0.0)$ & $1.000^{*}$ \\
\hline Stroke & $39(21.4)$ & $24(26.4)$ & $15(16.5)$ & 0.148 \\
\hline Sedentary lifestyle & $74(40.7)$ & $48(52.7)$ & $26(28.6)$ & 0.002 \\
\hline COPD & $14(7.7)$ & $9(9.9)$ & $5(5.5)$ & 0.404 \\
\hline Chronic Renal Insufficiency & $9(4.9)$ & $7(7.7)$ & $2(2.2)$ & $0.169 *$ \\
\hline Congestive heart failure & $11(6.0)$ & $8(8.8)$ & $3(3.3)$ & 0.213 \\
\hline Others & $46(25.3)$ & $27(29.7)$ & $19(20.9)$ & 0.232 \\
\hline
\end{tabular}

\#to compare means: student t-test; to compare proportions: Pearson's chi-squared test ; ${ }^{*}$ Fisher's exact test; COPD: Chronic Obstructive Pulmonary Disease; Other: Dementia, Osteoporosis, Parkinson's, Alzheimer's, Depression, Neoplasms, Epilepsy, Malnutrition, Hypothyroidism, Epilepsy, Known Osteoporosis, Anterior Contralateral Fracture, Glaucoma, Pulmonary Hypertension, Alcoholism, Labyrinthitis, and Atrial Fibrillation.

Regarding morbidities, most of the elderly included in the study (87.4\%) had at least one morbidity. Hypertension was the most prevalent morbidity, followed by Diabetes Mellitus.

The other morbidities studied had an incidence lower than $10 \%$, both when evaluated throughout the sample and when stratified by groups. Other morbidities were identified in 46 elderly people $(25.3 \%)$, among which were: dementia (10), known osteoporosis (4), epilepsy (4), malnutrition (2), hypothyroidism (11), Parkinson's disease (5), Alzheimer's disease (9), pulmonary hypertension (2), alcoholism (2), glaucoma (2), depression (5), HIV (2), atrial fibrillation (1)), neoplasias (12), previous contralateral fracture labyrinthitis (2).

The group treated in the usual manner presented a greater number of morbidities than the group included in the program. As for the association with more than one morbidity, elderly persons with a greater number of morbidities were more common in both groups, and there was a higher prevalence of three or more morbidities in the usual treatment group.

Regarding the results of the program indicators, the data were analyzed for the entire group of patients included in the study (182) and then compared between the groups (table 2). There was an increase in medical visits, a small increase in the number of nutritional assessments and a significant increase in the approach and monitoring by social services of the patients included in the program. There was also an increase in the planning of hospital discharge from the first day of admission of the patients included in the program $(p<0.001)$. There was no further mechanical restraint following the implementation of the program. 
Table 2. Results of indicators/guidelines established by program. Porto Alegre, Rio Grande do Sul, 2016.

\begin{tabular}{|c|c|c|c|c|}
\hline & $\begin{array}{l}\text { Total } \\
(n=182)\end{array}$ & $\begin{array}{l}\text { Usual treatment } \\
(n=91)\end{array}$ & $\begin{array}{l}\text { Included in program } \\
(\mathrm{n}=91)\end{array}$ & $p$ \\
\hline Variables & $\mathrm{n}(\%)$ & $\mathrm{n}(\%)$ & $\mathrm{n}(\%)$ & \\
\hline Onset of surgery (days) ${ }^{1}$ & $6(3-10)$ & $9(6-13)$ & $3(2-5)$ & $<0.001$ \\
\hline Beginning of surgery (bands) & & & & $<0.001$ \\
\hline Up to 2 days & $41(22.5)$ & $0(0.0)$ & $41(45.1)^{*}$ & \\
\hline 3 days & $17(9.3)$ & $0(0.0)$ & $17(18.7)^{*}$ & \\
\hline 4 days & $18(9.9)$ & $11(12.1)$ & $7(7.7)$ & \\
\hline 5 to 10 days & $63(34.6)$ & $43(47.3)^{*}$ & $20(22.0)$ & \\
\hline$>10$ days & $43(23.6)$ & $37(40.7)^{*}$ & $6(6.6)$ & \\
\hline \multicolumn{5}{|l|}{ Assessments } \\
\hline Visit of hospital doctor by $14.00^{1}$ & $4(2-5)$ & $3(2-5)$ & $4(3-6)$ & 0.007 \\
\hline Visit of orthopedist by $14.00^{1}$ & $5(4-7)$ & $5(4-6)$ & $6(4-7)$ & 0.066 \\
\hline Nutritionist Evaluation & $171(94.0)$ & $84(92.3)$ & 87 (95.6) & 0.534 \\
\hline Social Worker Evaluation & $51(28.0)$ & $14(15.4)$ & $37(40.7)$ & $<0.001$ \\
\hline Pain & $175(96.2)$ & $86(94.5)$ & $89(97.8)$ & $0.444^{\mathrm{a}}$ \\
\hline Discharge plan on day 1 & $63(34.6)$ & $0(0.0)$ & $63(69.2)$ & $<0.001$ \\
\hline Mechanical Restraint & $6(3.3)$ & $6(6.6)$ & $0(0.0)$ & $0.029^{a}$ \\
\hline Early withdrawal of urinary catheter & $81(44.5)$ & $14(15.4)$ & $67(73.6)$ & $<0.001$ \\
\hline Use of Prophylactic ATB & $170(93.4)$ & $81(89.0)$ & $89(97.8)$ & $0.037^{\mathrm{a}}$ \\
\hline Morbidities & $159(87.4)$ & $80(87.9)$ & $79(86.8)$ & 1.000 \\
\hline Death & $13(7.1)$ & $11(12.1)$ & $2(2.2)$ & 0.021 \\
\hline Readmission within 30 days & $7(3.8)$ & $4(4.4)$ & $3(3.3)$ & 1.000 \\
\hline Days of hospitalization & $11(7-17)$ & $16(12-21)$ & $7(5-10)$ & $<0.001$ \\
\hline Bands of days of hospitalization & & & & $<0.001$ \\
\hline$\leq 5$ & $26(14.3)$ & $0(0.0)$ & $26(28.6)^{*}$ & \\
\hline 6 to 10 & $64(35.2)$ & $18(19.8)$ & $46(50.5)^{*}$ & \\
\hline 11 to 15 & $36(19.8)$ & $26(28.6)^{*}$ & $10(11.0)$ & \\
\hline$>15$ & $56(30.8)$ & $47(51.6)^{*}$ & $9(9.9)$ & \\
\hline Total cost (reais)** & $\begin{array}{l}8.295 \\
(5.825-11.042)\end{array}$ & $\begin{array}{l}10.520 \\
(8.351-14.557)\end{array}$ & $\begin{array}{l}5.900 \\
(4.981-7.448)\end{array}$ & $<0.001$ \\
\hline Total (dollars)** & $\begin{array}{l}2.291 \\
(1.638-3.142)\end{array}$ & $\begin{array}{l}2.942 \\
(2.308-4.123)\end{array}$ & $\begin{array}{l}1.683 \\
(1.399-2.154)\end{array}$ & $<0.001$ \\
\hline
\end{tabular}

*statistically significant association by residuals test adjusted to 5\% significance; ${ }^{* *}$ Median (P25-P75); \# to compare medians: Mann-Whitney test; to compare proportions: Pearson chi-squared test ; ${ }^{a}$ Fisher's exact test.

The use of prophylactic antibiotics and early withdrawal of the urinary catheter was significantly higher in the group included in the program $(p<0,001)$.

There was a significant difference in the time elapsed between the fracture and the beginning of the surgery $(p<0.001)$. Usual treatment patients waited for an average of nine days, while the patients included in the program waited three days on average. When stratified by time bands, there was also a significant difference between groups. In the usual treatment group, all 91 patients $(100 \%)$ waited more than four days. In the group of patients included in the program, $58(63.7 \%)$ elderly persons were surgically treated within a maximum of three days (figure 1). 


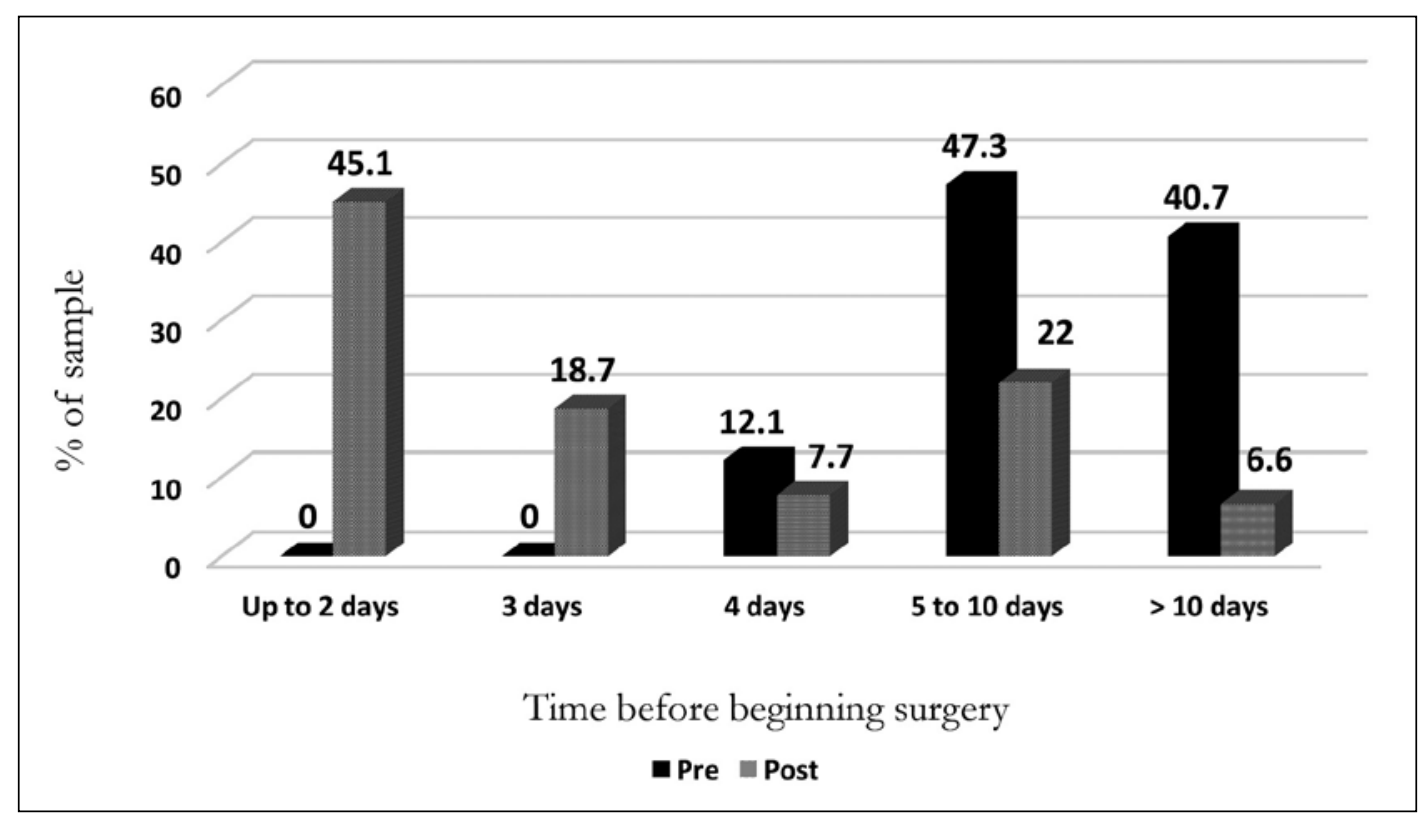

Figure 1. Comparison of times before beginning surgery before and after the program. Porto Alegre, Rio Grande do Sul, 2016.

The time before beginning surgery was a determining factor for the average hospital stay ( $p<0.001)$. The longer the time required to begin surgery, the longer the hospitalization (figure 2). The study identified the determinants for noncompliance as a goal of 48 hours (two days) to begin surgery after the start of the program. The "weekend effect" was the main factor. Patients hospitalized on these days encounter a different hospital resource structure, with changes in the rosters of nursing, anesthesiologist and clinical teams, with the latter on on-call schedules (without doctors performing their normal rounds), as well as logistic problems. The readmission in 30 days variable did not present a significant difference between the groups of patients. There was a reduction in the rate of in-hospital deaths from $11(12.1 \%)$ in the usual treatment group to two $(2.2 \%)$ in the program group $(p<0.001)$. There was also a reduction in mean hospital stay between the two groups, from 16 (12-21) days, to seven (5-10) days $(p<0.001)$ in the program group. When stratified into bands of days of hospitalization, all the bands were statistically significant in the adjusted residuals test at $5 \%$. There was a reduction in long-stay patients
(> 15 days), from $47(51.6 \%)$ before the program to nine $(9.9 \%)$ after the program.

There was also a reduction in the number of postoperative complications, from $24(26.4 \%)$ to $13(14.4 \%)$. Urinary tract infections significantly declined, from $13(14.3 \%)$ in the usual treatment group to $0(0.0 \%)$ in the program group $(p<0.001)$.

In the analysis of the costs, the treatments after the application of the program were significantly less costly ( $\mathrm{p}<0.001)$, as the usual treatment patients used the ICU (Intensive Care Unit) more than the units included in the program. In the comparison between the groups, the costs of the usual treatment group were $\mathrm{R} \$ 10,520.00$ (US $\$ 2,942$ ) on average and those of the group included in the program were $\mathrm{R} \$ 5,900.00$ (US\$1,683) on average. In the analysis of the overall costs (182 patients), the hospital had expenses of $\mathrm{R} \$ 1,860,140.00$ (US $\$ 543,900)$, with $\mathrm{R} \$ 1,250,971.72$ (US\$365,781) spent on the usual treatment group and R\$609,188.68 (US\$178,125) spent on the group included in the program, a ratio of $67.3 \%$ to $32.7 \%$ of the total costs, respectively. Some factors were identified as determinants for 
the composition of overall cost, including the time before beginning surgery (figure 3). The shorter the time before surgery, the lower the costs $(p<0.001)$. The same was true for days of hospitalization and number of morbidities, with the shorter the length of stay, the lower the cost of treatment $(p<0.001)$. The number of morbidities was a determining factor for longer mean permanence $(p<0.001)$, with the higher the morbidity values, the more days spent in hospital $(p<0.001)$.

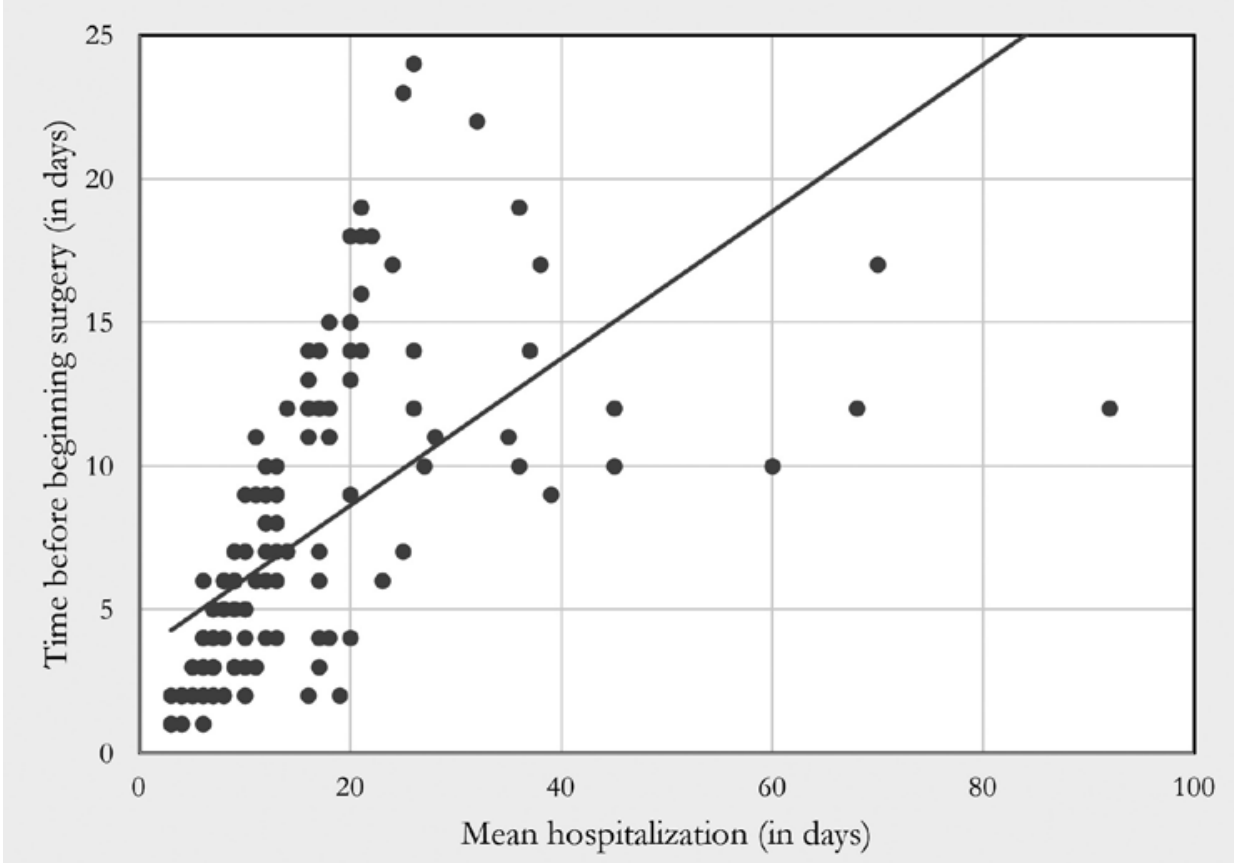

Figure 2. Analysis of impact of time before beginning surgery on length of hospitalization. Porto Alegre, Rio Grande do Sul, 2016.

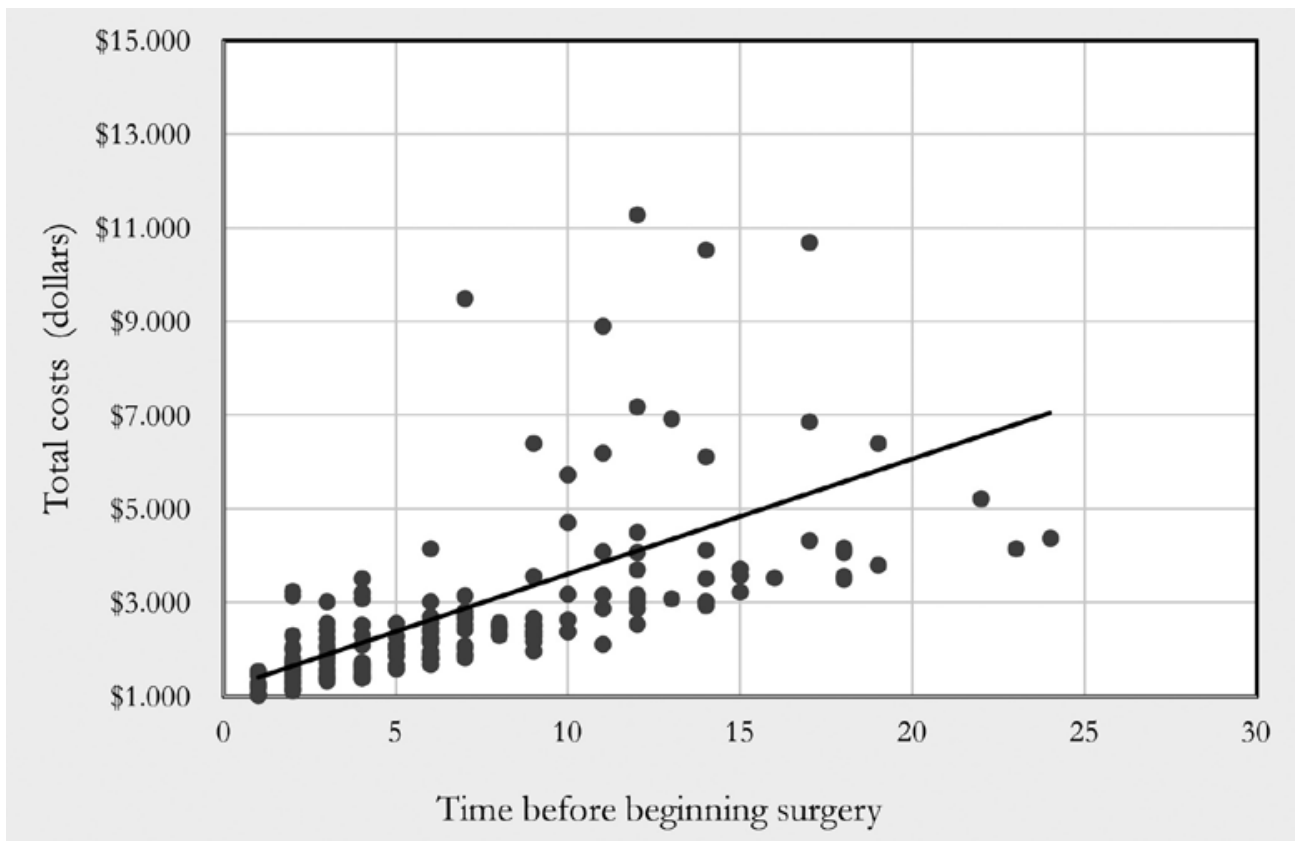

Figure 3. Relationship between time before beginning surgery and total costs. Porto Alegre, Rio Grande do Sul, 2016. 
The largest hospital bills were attributed to patients with a longer time interval between the fracture and the onset of surgery, more than seven days of hospitalization, more than two morbidities and complications in the postoperative period.

The most prevalent complications were delirium, renal insufficiency and urinary tract infection, followed by pneumonia and surgical site infection. However, there was no statistical association between the number of complications and mean permanence. Age was not related to higher costs ( $p>0.05$ ). The mean age of the group of patients with the highest costs was the same as that of the entire surveyed group.

\section{DISCUSSION}

The present study revealed a greater prevalence of females than males. Similar results were found in studies published in the databases of the American Academy of Orthopedic Surgeons (AAOS) ${ }^{10}$, the British Orthopaedic Association (BOA) ${ }^{7}$ and the Brazilian Ministry of Health ${ }^{11}$.

According to Barros et al. ${ }^{12}$ and Eriksson et al. ${ }^{4}$ the higher prevalence of hip fractures among women may be related to the fact that females have a longer life expectancy and are therefore more exposed to risk factors. They also initiate the process of bone mass decline before men and are more susceptible to falls, in addition to anthropometric influences and genetic factors related to gender.

Longer-lived elderly persons (between the seventh and eight decade of life) were the most affected. Hungria et al. ${ }^{13}$, Gibson and Hay ${ }^{14}$ and Boulton et al. ${ }^{15}$ argue that the proximal fracture of the femur affects older elderly people, as the musculoskeletal alterations that compromise the performance of motor skills make it difficult for such individuals to adapt to the environment and predispose them to falls from their own height.

In spite of the general morbidity rate and the difference in the morbidity profile found among the groups studied, there was no prevalence of a single type of morbidity. Mesquita et al. ${ }^{16}$ described a higher prevalence of heart disease, while Uliana et al. ${ }^{17}$ states that the prevalence of morbidities is high among hospitalized patients with hip fractures and that there is a high risk for complications.

The overall mortality rate was $7.1 \%$, consistent with the results found by Thomas et al. ${ }^{18}$ and Gilberg et al. ${ }^{19}$, which were between $8.5 \%$ and $6.5 \%$, respectively, as well as those of Tarrant et al. ${ }^{20}$. However, in the present study, mortality decreased after the application of the program, corroborating the "principles of comanagement" advocated and applied by Friedman. The average length of hospitalization decreased significantly after the application of the program. However, length of stay was still longer than those obtained by Friedman et al. ${ }^{5}$.

The studies and experiences validated by Mendelson and Friedman ${ }^{8}$ show that early surgery is an important and determinant factor for the improvement of the quality of treatment, including in terms of cost effectiveness. After the start of the program, the average time between the fracture and the beginning of surgery diminished from nine to three days $(p<0.001)$. The guidelines of the American Academy of Orthopaedic Surgeons (AAOS) $)^{10}$ and the British Orthopaedic Association (BOA) ${ }^{7}$ recommend that surgery is performed on the same day or within 24 hours. However, the population analyzed in the present study is served by a health network involving more than one institution, separated by a distance of kilometers and with different teams, and so the goal was adjusted to 48 hours (two days). In this context, the results of the program were very satisfactory, with a reduction of $70 \%$ in the time required to begin surgery, although not all patients started surgery in the anticipated period. The main difficulty faced in meeting the two-day target was the "weekend effect", when there are changes in the staffing and resource structure of the hospital. In an extensive study with 2989 patients by Thomas et al. ${ }^{18}$, the socalled weekend effect meant that in addition to delays at the beginning of surgery, there was an increase in mortality rates in the first 30 days after fractures in patients hospitalized at weekends.

The program also displayed better results in terms of procedural goals. There was an increase in the use of prophylactic antibiotics and, in parallel, a reduction in surgical site infections. While it was not possible to establish a direct relationship between the two variables due to the small number of cases, 
studies by Gonçalves et al. ${ }^{21}$ and Machado et al. ${ }^{22}$ describe prophylactic antibiotics as one of the main strategies for preventing surgical site infections. These studies demonstrate that the use of such antibiotics, combined with general measures of surgical infection prevention, have been shown to be effective when performed in a rational manner.

Bouvet et al. ${ }^{23}$ Rizk et al. ${ }^{24}$ and Rennke and Ranji $1^{25}$ state that urinary tract infection is the first major cause of nosocomial infection and therefore recommend avoiding a urinary catheter, or, when its use is necessary, removing it as quickly as possible. Similar findings were obtained in the present study, which demonstrated a significant association between the early withdrawal of the urinary catheter and a reduction in the percentage of urinary tract infections for the group included in the program.

Although mechanical restraints were not used on any patients during the application of the program, there was a reduction in cases of delirium in this group. Some studies, such as that of Bracco et al, ${ }^{25}$ indicate that delirium may be due in part to the functional decline generated by physiological stress caused by the long periods of hospitalization ${ }^{25}$ combined with morbidities. The patients included in the program had a shorter wait before starting surgery and consequently reduced their mean hospitalization time, which may explain the reduction of delirium in this group.

The $\mathrm{AAOS}^{10}$ and $\mathrm{BOA}^{7}$ contemplate a collaborative model of care that presupposes more frequent communication, anticipates complications and avoids iatrogenic events. When applied in the program of this study, such measures demonstrated favorable results, with the increased presence of the hospital doctor in the management of the cases $(p=0.007)$. Social workers were incorporated into the model and also significantly increased their role in the management of the cases ( $p<0.001)$, working as "managers" in preparation for hospital discharge from day one. According to Bracco et al. ${ }^{26}$ this measure is highly relevant as the preparation of the patient and their family and the evaluation of the conditions of the home environment and care structure after discharge avoids readmissions and contributes to the quality of life and rehabilitation of elderly persons with hip fractures.
The final analysis contained the costs involved in the treatment of hip fractures, which fell by $44 \%$ following the start of the program. The costdetermining factors, besides the direct costs involved in treatment, were the time required to start surgery, the length of hospital stay and the total number of morbidities.

There is some complexity involved when comparing cost evaluation results with those of other studies. The form of appropriation of costs differs from one institution to another and not all determining factors are investigated. In the study by Bracco et al. ${ }^{26}$, the same cost appropriation measures were used, and an average cost of hospitalization of $\mathrm{R} \$ 8,266.25$ (US\$2,417) was recorded, a little lower than the present study. However, it is unclear whether ICU stays were included, and morbidity profile data or spending on other cost-determining factors that allow comparisons were not reported. Although common in Brazil, studies of the costs associated with hip fractures mostly provide results focused on the costs to the remuneration system, based on data from the sources of payment (SIH/ SUS and ANS). ${ }^{27,28}$ According to Gerard et al. ${ }^{29}$ these costs do not reflect the costs of care, but rather the payments made to health institutions, although they consider the costs of the initial (hospital) treatment, which is the most costly and is also definitive of other costs, and the money spent on rehabilitation and/or readmissions. Studies are often follow-ups (from 1 to 2 years), which do not allow comparisons. Furthermore, when comparing studies from other countries it is necessary to consider the differences in the approach to and the organization of treatments, since the average costs of hospitalizations are much higher than in Brazil. This increase most likely corresponds to the costs of geriatric rehabilitation units as a second stage of hospitalization, which is not a common practice in Brazil. A study by Burgers et al. ${ }^{6}$ in the Netherlands identified an average cost of $€ 5,732$, while a study by Ginsberg et al. ${ }^{21}$ in the US found an average hospitalization cost of (US\$7,826.00) ${ }^{20}$. These studies agree that the early beginning of surgery and unnecessary days of hospitalization are key factors.

In the present study, the highest costs were related to direct and daily stay costs. The patients in the 
usual treatment group used the ICU more and had a $70 \%$ increase in average costs of the daily stay type.

The variables tested in this study were based on models and experiences obtained in other countries, adapted to the reality of a health network of the Brazilian Unified Health System (SUS). The sample of 182 patients is perhaps not highly representative of the universe of elderly victims of falls with hip fractures. However, even with the model adapted to the described reality, the results were the same as those reported by Ginsberg et al. ${ }^{19}$, Thomas et al. ${ }^{28}$ and Caillet et al. ${ }^{30}$ in their social contexts, with a significant association between the application of the program and the reduction of waiting time required to begin surgery, average length of stay, mortality rates and hospital costs. Follow-up studies should be aimed at further analysis.

\section{CONCLUSIONS}

The program of care for elderly persons with hip fractures, applied in a network structure, modified the expected results by reducing mortality, mean

\section{REFERENCES}

1. Instituto Brasileiro de Geografia e Estatística. Pesquisa nacional de saúde 2013: percepção do estado de saúde, estilos de vida e doenças crônicas Brasil, grandes regiões e unidades da federação. Ministério da saúde. Rio de Janeiro: IBGE; 2014.

2. Auerbach AD, Kripalani S, Vasilevskis EE, Sehgal N, Lindenauer PK, Metlay JP, et al. Preventability and causes of readmissions in a national cohort of general medicine patients. JAMA Intern Med. 2016;176(4):484-93.

3. Tsang C, Cromwell D. Statistical methods developed for the National Hip Fracture Database annual report, 2014: a technical report. London: Royal College of Surgeons of England; 2014.

4. Eriksson M, Kelly-Pettersson P, Stark A, Ekman AK, SkoIdenberg O. 'Straight to bed' for hipfracture patients: a prospective observational cohort study of two fast-track systems in 415 hips. Injury. 2012;43(12):2126-31. hospital stay, postoperative complications and treatment costs during hospitalization.

The strategies adopted, using validated clinical guidelines, protocols and tools for care network management, solved problems diagnosed before its application, providing more effective and efficient results for the care of the elderly included in the program.

The collaborative model allowed more coordinated assistance, with more effective planning of the initial approach to the elderly and consequent preparation for hospital discharge. For this to be possible, managerial and technological tools were fundamental for the team to improve its communication efficiency.

In realities where resources are scarce, such as the Unified Health System (SUS), the program linked health care management to clinical guidelines and proved to be cost-effective and capable of bringing together teams to achieve superior outcomes for patients, the teams themselves, hospital institutions and public administrators.
5. Friedman SM, Mendelson DA, Bingham KW, Kates SL. Impact of a Comanaged Geriatric Fracture Center on Short-term Hip Fracture Outcomes. Arch Intern Med. 2009;169(18):1712-7.

6. Burgers PT, Hoogendoorn M, Van woensel EA, Poolman RW, Bhandari M, Patka P, et al. Total medical costs of treating femoral neck fracture patients with hemi-or total hip arthroplasty: a cost analysis of a multicenter prospective study. Osteoporosis Int. 2016;27(6):1999-2008.

7. British Orthopaedic Association. More patients surviving due to continued improvements in hip fracture care. Brighton: Medical News Today; 2014.

8. Mendelson DA, Friedman SM. Principles of comanagement and the geriatric fracture center. Clin Geriatr Med. 2014;30(2):183-9.

9. Porter ME, Teisberg EO. Repensando a saúde: estratégias para melhorar a qualidade e reduzir custos. Porto Alegre: Bookman; 2007. 
10. American Academy of Orthopaedic Surgeons. Management of hip fractures in the elderly. evidence - based clinical practice guideline. Rosemont: AAOS; 2014.

11. Ministério da Saúde. Departamento de Ciência e Tecnologia, Secretaria de Ciência, Tecnologia e Insumos Estratégico. ELSA Brasil: maior estudo epidemiológico da América Latina. Rev Saúde Pública. 2009;43(1):1-2.

12. Barros IFO, Pereira MB, Weiller TH, Anversa ETR. Internações hospitalares por quedas em idosos brasileiros e os custos correspondentes no âmbito do Sistema Único de Saúde. Rev Kairós. 2015;18(4):63-80.

13. Hungria Neto JS, Dias CR, Almeida JDB. Características epidemiológicas e causas da fratura do terço proximal do fêmur em idosos. Rev Bras Ortop. 2011;46(6):660-7.

14. Gibson AA, Hay AW, Ray DC. Patients with hip fracture admitted to critical care: epidemiology, interventions, and outcome. Injury. 2014;45(7):1066-70.

15. National Hip Fracture Database. Falls and Fragility Fracture Audit Programme (FFFAP). London: Royal College of Physicians; 2014.

16. Mesquita GV, Lima MALTA, Santos AMR, Alves ELM, Brito JNPO, Martins MCC. Morbimortalidade em idosos por fratura proximal do fêmur. Texto Contexto Enferm. 2009;18(1):67-73.

17. Uliana CS, Abagge M, Malafaia O, Kalil Filho FA, Cunha AM. Fraturas transtrocantéricas: avaliação dos dados da admissão à alta hospitalar. Rev Bras Ortop. 2014;49(2):121-8.

18. Thomas CJ, Smith RP, Uzoigwe CE, Braybrooke JR. The weekend effect. short-term mortality following admission with a hip fracture. Bone Joint J. 2014;96B(3):373-8.

19. Ginsberg G, Adunsky A, Rasooly I. A cost-utility analysis of a comprehensive orthogeriatric care for hip fracture patients, compared with standard of care treatment. Hip Int. 2013;23(6):570-5.

20. Tarrant SM, Hardy BM, Byth PL, Brown, TL, Attia j, Balogh, ZJ. Preventable mortality in geriatric hip fracture. Bone Joint J. 2014;96B(9):1178-84.

21. Gonçalves MLM, Silva MJS, Torres RM. Antibioticoprofilaxia e tratamento de infecção do sítio cirúrgico na artroplastia de quadril. Rev Bras Farm Hosp Serv Saúde São Paulo. 2014;5(3)18-22.
22. Machado A, Ferraz AAB, Ferraz E, Arruda E, Nobre J, Konkewicz LR, et al. Prevenção da infecção hospitalar [Internet]. [Sem Local]: Sociedade Brasileira de Infectologia; 2016 [acesso em 22 fev. 2016]. Disponível em: https:// www.researchgate.net/profile/Alvaro_Ferraz2/ publication/237238421_Sociedade_Brasileira_de_ Infectologia/links/5469daf40cf2397f782dd210.pdf

23. Bouvet C, Lubbeke A, Bandi C, Pagani L, Stern $\mathrm{R}$, Hoffmeyer $\mathrm{P}$, et al. Is there any benefit in preoperative urinary analysis befor elective total joint replacement? Bone Joint J. 2014;96B(3):390-4.

24. Rizk P, Morris W, Oladeji P, Huo M. Review of postoperative delirium in geriatric patients undergoing hip surgery. Geriatr Orthop Surg Rehabil. 2016;7(2):100-5.

25. Rennke S, Ranji SR. Transitional care strategies from hospital to home: a review for the neurohospitalist. Neurohospitalist. 2015;5(1):35-42.

26. Bracco OL, Fortes EM, Raffaelli MP, Araújo DV, Santili C, Castro ML. Custo hospitalar para tratamento da fratura aguda do fêmur por osteoporose em dois hospitais-escola conveniados ao Sistema Único de Saúde. São Paulo: Escola Paulista de Medicina; 2008.

27. Brasil. Ministério da Saúde, DATASUS. Sistema de informações hospitalares - SIH/SUS. Notas técnicas [Internet]. Rio de Janeiro: DATASUS; 2016 [acesso em 22 fev. 2016]. Disponível em: http://tabnet. datasus.gov.br/cgi/sih/midescr.htm

28. Brasil. Agência Nacional de Saúde Suplementar. Padrão para Troca de Informação de Saúde Suplementar - TISS [Internet]. Rio de Janeiro: ANS; 2016. [acesso em 22 fev. 2016]. Disponível em: http://www.ans.gov.br/prestadores/tiss-trocade-informacao-de-saude-suplementar\#sthash. K82m8AlO.dpuf

29. La Forgia GM, Couttolenc BF. Desempenho hospitalar no Brasil: em busca da excelência. São Paulo: Singular; 2009.

30. Caillet P, Klemm S, Ducher M, Aussem A, Schott AM. Hip fracture in the elderly: a re-analysis of the EPIDOS study with causal bayesian networks. Plos One. 2015;10(3):1-8. 